\title{
OPTIMIZATION OF 35-35 INTERNALLY AND EXTERNALLY LOADED CS-TPA ON SBA 15 CATALYST SYSTEM FOR THE PRODUCTION OF BIODIESEL FROM WASTE COOKING OIL
}

\author{
Anitha.A. ${ }^{1}$, Abbas Mohaideen.J. ${ }^{2}$, Dawn ${ }^{3}$.S.S and Revathy \\ ${ }^{1,3}$ Department of Chemical engineering,Sathyabama University, Chennai, India \\ ${ }^{2}$ Principal, Mamallan Institute of Technology, Chennai, India \\ Email: arniish@yahoo.com
}

\begin{abstract}
The demand for automobile fuels is increasing every day which may be attributed to the depleting non-renewable energy resources. This has necessitated the need for an alternate fuel. Biodiesel is a cleaner burning fuel than diesel and is a suiTable- replacement which is produced from raw materials that are non-toxic, biodegradable and renewable. Alkali catalyzed transesterification is considered to be the best amongst all methods available for the production of Biodiesel from oil. Owing to the corrosion and re usability problems associated with the alkali catalysts like $\mathrm{NaOH}$ and $\mathrm{KOH}$ a novel Heteropolyacid (HPA) catalyst system, 35-35 Internally and Externally loaded Cs-TPA on SBA 15 was synthesized. A process for the production of Biodiesel from waste cooking oil has been studied and the reaction conditions that affect the yield and purity of product esters like the quantity of the catalyst and methanol, the reaction time and temperature were optimized. Kinetics of the reaction were compared with that of the conventional alkali catalysts and it was found that the proposed heteropolyacid catalyst system gave higher yields. The catalyst activity did not vary much even after reusing the recovered catalyst system up to six runs. The specific gravity, viscosity and Flash point for the waste cooking oil ester produced were measured and is found to be as per the ASTM standards.
\end{abstract}

KEY WORDS: Transesterification, Biodiesel, Waste Cooking Oil, Kinetics, HPA.

\section{INTRODUCTION}

Environmental concerns and need of fossil fuel preservation for future generation has necessitated a methodology for production of alternate fuels. Bio-diesel presents a suiTable- renewable substitute for petroleum based diesel. The most widely opted method for the production of biodiesel is by transesterification, which refers to a catalyzed chemical reaction involving vegeTable- oil and an alcohol to yield fatty acid alkyl esters (i.e., biodiesel) and glycerol. Triglycerides as the main component of vegeTable- oil consist of three long chain fatty acids esterified to a glycerol backbone. When triglycerides react with an alcohol (e.g., methanol), the three fatty acid chains are released from the glycerol skeleton and combine with the alcohol to yield fatty acid alkyl esters .Glycerol is produced as a by-product. Methanol is the most commonly used alcohol because of its low cost and is the alcohol of choice in the processes developed in this study. In general, a large excess of methanol is used to shift the equilibrium far to the right.

Numerous studies have been conducted on biodiesel production and emission testing in the past two decades [1]. Most of the current challenges are targeted to reduce its production cost, as the cost of biodiesel is still higher than its petro-diesel counterpart. This opens a golden opportunity for the use of waste or recycled oils as its production feedstock. Everywhere in the world, there is an enormous amount of waste lipids generated from restaurants, food processing industries and fast food shops everyday. Reusing of these waste greases can not only reduce the burden of the government in disposing the waste, maintaining public sewers and treating the oily wastewater, but also lower the production cost of biodiesel significantly [1].

The typical acids and bases employed for transesterification include Sulfuric, Phosphoric, Hydrochloric, Organic Sulfonic acids and $\mathrm{NaOH}, \mathrm{KOH}$ and Carbonates. Though the homogeneous base catalyzed transesterification reaction is faster then the acid catalyzed reaction, the main disadvantages include energy intensiveness, difficulty in separating the glycerol from methyl esters. Apart from that when acids like Sulfuric acid are used they exhibit a lot of corrosion problems, adding to the maintenance cost in Biodiesel Production. Water tolerant solid acid catalysts can replace strong liquid acids thereby eliminating the corrosion problems associated with their use and consequent environmental hazards posed by them. In the search of water tolerant acid catalysts, Heterolpoly acid (HPA) appear to be the most appropriate choice. Most of these systems have acidity in the range of superacids with the possibility of tailoring the porous architecture as well as solubility in water (like the Cs salt).

Heteroployacids particularly the Keggin type 12Tungstophosphoric acid (TPA) exhibits high acidic strengths and have performed admirably in a wide variety of acid catalyzed reactions[2,3,4]. However TPA suffers from drawbacks such as very low surface area which hinders accessibility to the strong acidic sites whereas high affinity for polar media renders it soluble. The Cesium salts of TPA are characterized by higher surface area and 
resistance to leaching in polar media. Hence Cesium substituted Tungstophosphoric acid (Cs-TPA) has been widely used. Several supports such as silica gel, Alumina, Zirconia, Carbon and Mesoporous silica have been used to enhance the dispersion of TPA, thereby increasing the accessibility to their acid sites while lowering their solubility in polar media. Silica has been widely favored as a supporting material for Cs-TPA since it interacts weakly with the Keggin anions and thus preserves their structure. $A$ larger pore diameter $(7-9 \mathrm{~nm})$ and comparable internal surface of SBA - 15 relative to $\mathrm{MCM}-41$ enables higher loading of catalytic phase with little pore blockage and improved control of its location. Hence SBA- 15 is a proper support for Cs-TPA [5,6,7].

In the present work SBA-15 was employed as a support for preparation of high loading composite Cs TPA on the external surface and inside the channels. The catalytic activity of the novel catalyst was tested and optimum conditions were determined for the transesterification of waste cooking oil to produce methyl ester (Biodiesel ) .The specific gravity, viscosity and Flash point of the obtained biodiesel were found comparable with the standards for Bio Diesel based on American Society for Testing and Materials. The variables were a) amount of catalyst, b) amount of Methanol to oil ratio, c) temperature of the reaction and d) reaction time. Kinetic studies were done to study the progress of the reaction for the optimized reaction time.

\section{EXPERIMENTAL PROCEDURE}

\section{A. Preparation of Catalyst system}

SBA-15 was prepared according to the procedure presented in [8] by crystallization from acidic solutions of Poly (Ethylene glycol) - block - Poly (Propylene glycol) block - Poly (Ethylene glycol) copolymer (Aldrich) and TEOS (Aldrich). The composite with high loading of Cs-TPA on the external surface and inside the channels of SBA-15 was prepared according to the procedure described in [5].

\section{B. Reaction Procedure}

Transesterification reactions were carried out in a 250 $\mathrm{ml}$ glass reactor with a condensor. The reaction procedure was as follows. First, a known quantity of the catalyst system (Cs-TPA 35-35 Internally and Externally loaded on SBA-15) was dispersed in Methanol under magnetic stirring. Then Waste Cooking Oil (WCO) in the molar ratio of 6:1, methanol to oil was added to the mixture and heated to about $60 \cdot \mathrm{C}$. The reaction was allowed to take place for two hours. Once the reaction is over the two phase product formed as a result of transesterification was separated using a separating funnel. Upper layer consists of biodiesel, alcohol and some soap (formed as result of side reaction saponification - free fatty acids get converted to soap). Lower layer consists of Glycerin, excess alcohol, Catalyst, impurities, and traces of unreacted oil. Purification of the upper layer was done by washing with warm water. As water is immiscible with Biodiesel it can easily be separated from biodiesel. Experiments were repeated to optimize the amount of catalyst, methanol to oil molar ratio, reaction time and temperature.

Kinetic studies were done for the optimized parameters to study the progress of the reaction. The catalyst in the lower phase was separated by centrifugation. Experiments were also conducted to test the reusability of catalyst for consistent yield.

\section{RESULTS AND DISCUSSION}

\section{A. Effect of amount of Catalyst on Biodiesel Yield}

In this study the amount of Cs-TPA 35-35 Internally and Externally loaded on SBA -15 was varied from 0.1 to 0.9 grams. The biodiesel yield improved with increasing catalyst addition and the maximum biodiesel yield was obtained by adding 0.75 grams of catalyst system which reached $88.9 \%$ in $2 \mathrm{hrs}$ as shown in Fig.1. However the yield was only $85.2 \%$ when about 0.9 grams of catalyst was added.

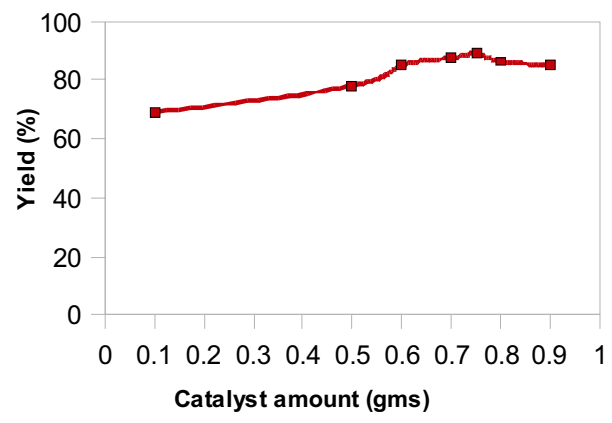

Fig.1. Optimization of Catalyst amount

WCO : 25gm; MeOH : 19.2 Time : 2hrs; Temp: $60^{\circ} \mathrm{C}$

\section{B. Effect of amount of Methanol on Biodiesel Yield}

The stoichiometry of this reaction requires three moles methanol per mole triglyceride to yield three moles Biodiesel and one mole glycerol. The effect of amount of methanol on biodiesel yield is illustrated in Fig.2 which shows that the reaction rate increased when the methanol quantity increased from $18 \mathrm{gms}$ to $24 \mathrm{grams}$. The biodiesel yield increased from $84.6 \%$ to $89.6 \%$. However when the amount of methanol was over $22 \mathrm{gms}$, glycerol separation became more difficult thus decreasing the Biodiesel yield. Based on this, the optimum methanol quantity is found to be $21 \mathrm{gms}$, which when calculated in terms of methanol to oil molar ratio is found to be 5.3:1. 


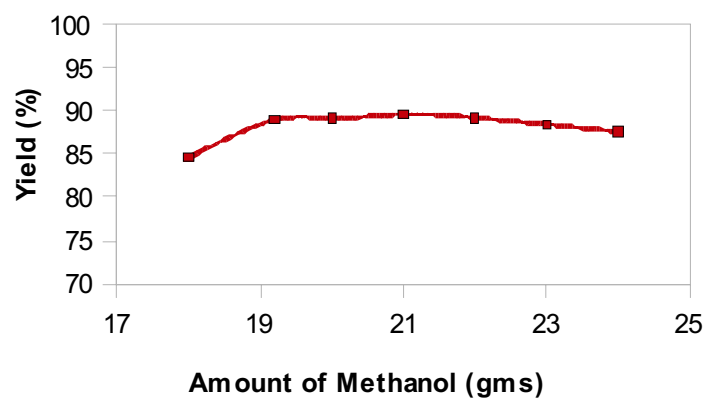

Fig.2.Optimization of Methanol Amount

WCO : 25; Cs-TPA/SBA- $15: 0.75 \mathrm{gm}$;

Time ; $2 \mathrm{hrs} ; \mathrm{Temp}: 60^{\circ} \mathrm{C}$

\section{Effect of Reaction time on Biodiesel Yield}

In the present work the reaction time was varied from 15 mins to 120 mins. The effect of reaction time on Biodiesel yield is shown in Fig.3. Based on the optimum yield of $90.5 \%$ the reaction time was fixed as 60 mins for the production of Biodiesel for the proposed catalyst system.

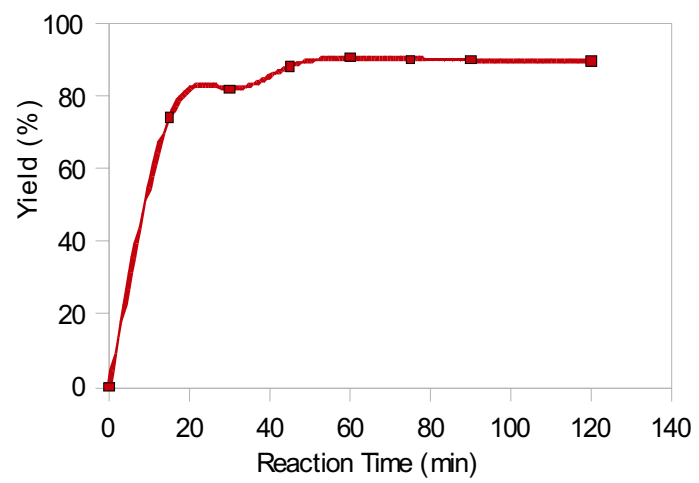

Fig .3.Optimization of Reaction Time

WCO :25 gm, MeOH : $21 \mathrm{gm}, \mathrm{Temp}: 60{ }^{\circ} \mathrm{C}$

\section{Effect of Reaction Temperature on Biodiese/ Yield}

Reaction temperature can influence the reaction rate and biodiesel yield. The reaction temperature was varied within a range from $40 \mathrm{deg}$ Celsius to $65 \mathrm{deg}$ Celsius, the effect of which is shown in Fig.4. The results indicate that the reaction rate was slow at low temperatures and the biodiesel yield was only $68 \%$ at 40 deg Celsius for $1 \mathrm{hr}$. the Biodiesel yield increased with increase of reaction temperature to $90.5 \%$ at a temperature of $60 \mathrm{deg}$ Celsius. The primary advantage of higher temperatures is a shorter reaction time. However if the reaction temperature exceeds the boiling range of Methanol, the Methanol will vaporize and form a large number of bubbles, which inhibit the reaction on the three phase inter phase. Therefore, the optimum reaction temperature for the transesterification of waste cooking oil to Biodiesel is considered to be around 60 deg Celsius.

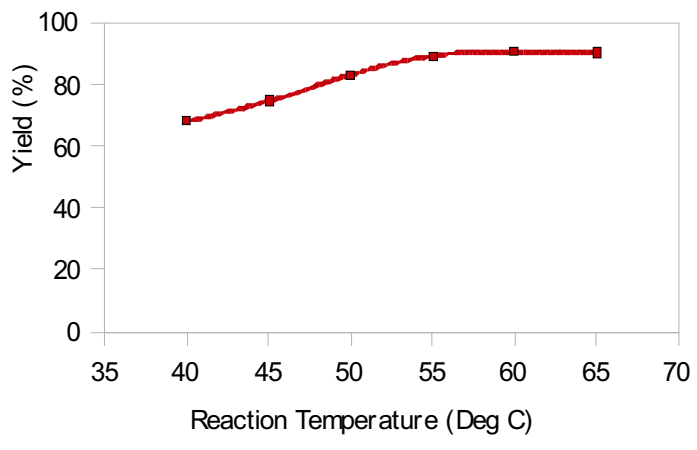

Fig. 4. Optimization of Reaction temperature $\mathrm{WCO}: 25 \mathrm{gm}, \mathrm{MeOH}: 21 \mathrm{gm}$, Time : $60 \mathrm{~min}$

\section{E. Kinetic Studies}

For every 10 mins of the optimized reaction time (60 mins) the yield of Biodiesel was calculated and shown in Fig. 5

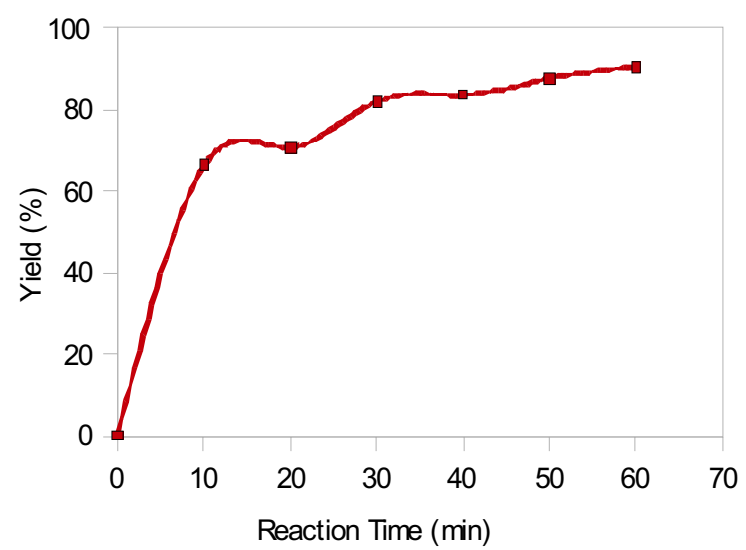

Fig. 5. Kinetic Studies

WCO: $25 \mathrm{gm}, \mathrm{MeOH}: 21 \mathrm{gm}$, Time : $60 \mathrm{~min}$, Temp : $60^{\circ} \mathrm{C}$

\section{F. Effect of Recycling of used catalyst on Biodiesel Yield}

After the reaction was complete the reaction mixture was centrifuged and separated to provide wet solid catalyst in order to recycle the catalyst, to study the catalyst life time and stability. To each sample fresh oil and Methanol were added as per the optimized conditions. Fig. 6 summarizes the experimental results which indicate that Cs-TPA 35-35 Internally and Externally loaded on SBA - 15 catalysed Biodiesel yields were similar to that of the maximum yield. The catalyst maintained sustained activity even after being used for 6 cycles and the Biodiesel yield decreased only slightly. The main reason resulting in the minor decrease in catalytic activity was due to the catalyst loss during the repeated experiments. From an economic point of view, the cost of the catalyst accounts for a large part of the cost of Biodiesel production. Therefore the stability and sustained activity of the catalyst are of great importance for industrial applications. Hence Cs-TPA 35-35 Internally and Externally loaded on SBA -15 can contribute much to 
decreasing the cost of Biodiesel production due to its long catalyst life time and good stability.

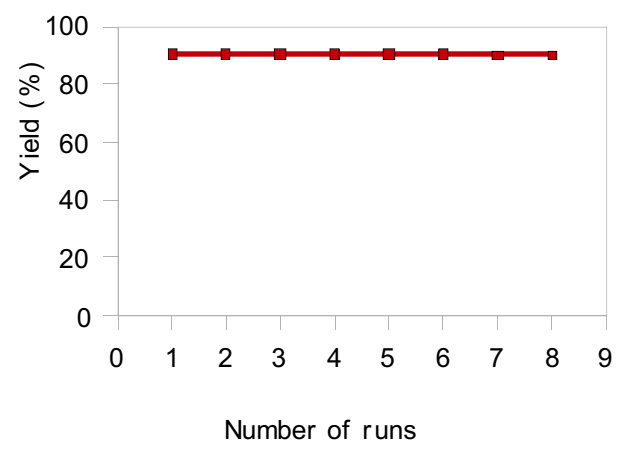

Fig .6. Reusability of Catalyst

\section{Comparison of Catalytic activity of the Cs-TPA 35- 35 Internally and Externally loaded on SBA -15 to Homogeneous Catalysts.}

A comparison of $\mathrm{NaOH}$ and $\mathrm{KOH}$ catalysts with CsTPA 35-35 Internally and Externally loaded on SBA -15 were carried out for the optimized conditions. The results illustrated in Fig. 7 indicate that for the same optimized reaction conditions the proposed catalyst system give a better yield in comparison with the conventional homogeneous catalysts.

\section{PHYSICAL PROPERTIES OF WASTE COOKING OIL ESTERS}

The specific gravity, viscosity at room temperature and the flash point of the waste cooking oil ester was found to be $0.88,4.2 \mathrm{cSt}$ and $70 \cdot \mathrm{C}$. The fuel properties of the obtained Biodiesel were found to be in reasonable agreement with the ASTM biodiesel standards.

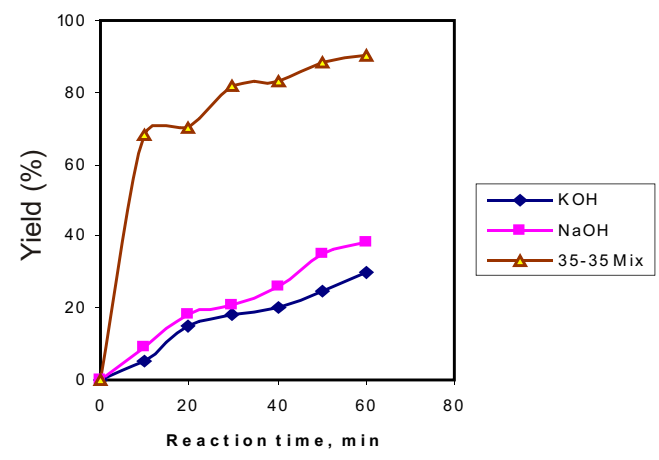

Fig 7. Com paris on of Catalyst Perform ance

\section{CONCLUSION}

The experimental results show that the supported solid acid catalyst demonstrated excellent catalytic activity, stability long catalyst life time. In the transesterification of WCO to Biodiesel, the biodiesel yield exceeded $90 \%$ for 60 mins. The catalyst also provided higher catalytic activity than homogeneous catalysts. For the best results economically the optimal conditions are $0.75 \mathrm{gms}$ supported catalyst, 5.3 : 1 molar ratio Methanol to WCO, reaction time of $60 \mathrm{~min}$ and temperature $60 \cdot \mathrm{C}$. Moreover the catalyst system can be reused for six times without much decrease in catalytic activity. As a solid acid catalyst, Cs-TPA 35-35 Internally and Externally loaded on SBA -15 decreases the cost of Biodiesel production and it has potential for industrial application in transesterification of WCO to Biodiesel.

\section{REFERENCES}

[1] Saifuddin N. and Chua, Malaysian Journal of Chemistry 6 (2004) 77.

[2] P Madhusudhan Rao, A.Wolfson, S. Kababya, S.Vega, M.V. Landau Journal of catalysis 232 (2005) 210.

[3] Y.Izumi, K. Urabe, M. Onaka, Zeolite, Clay and Heteropolyacid in Organic Reactions, VCH, Newyork, 1992, 99.

[4] M. Misono, Chem. Commun. (2001) 1141.

[5] P Madhusudhan Rao, M.V Landau, A. Wolfson, A.M Shapira- Tchelet, M.Herskowitz Microporous and Mesoporous materials 80 (2005) 43.

[6] L.R Pizzio, C.V. Caceres, M.N Blancu, Appl. Catal.A;Gen.167 (1998) 283.

[7] F. Marme, G. Coudurier, J. C. Vedrine, Micropor. Mesopor. Mater. 22 (1998) 151.

[8] D.Zhao, J.Sun, Q. Li, G. D. Stucky, Chem. Mater. $12(2000) 275$.

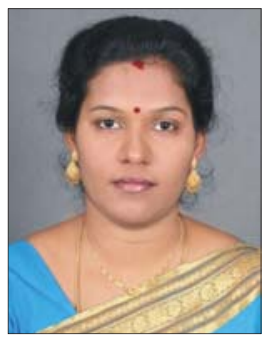

Ms. A.Anitha is working as a Senior Lecturer in the Department of Chemical Engineering ,Sathyabama University. Her areas of research interest are Catalysis and Bio fuels. 